\title{
Biodegradable Hydrogels Based on Alginate For Control Drug Delivery Systems
}

\section{MOHAMMAD SADEGHI*, FATEMEH SHAFIEI, ESMAT MOHAMMADINASAB, LALEH MANSOURI and MOHAMMAD JAVAD KHODABAKHSHI}

\author{
Department of Chemistry, Science Faculty, Islamic Azad University, Arak Branch, Arak, Iran.
}

(Received: January 24, 2014; Accepted: March 04, 2014)

http://dx.doi.org/10.12944/CWE.9.1.16

\begin{abstract}
In this work, synthesis and swelling behavior of a super absorbent hydrogel based on alginate and polyacrylamide (PAAm) was investigated. the structure of the product was established using FTIR and SEM spectroscopies. The alginate-polyacrylamide hydrogel exhibited a $\mathrm{pH}$ responsive swelling-deswelling behavior at pH's 3 and 9. This on-off switching behavior provides the hydrogel with the potential to control delivery of bioactive agents.
\end{abstract}

Key words: Alginate, Acrylamide, Hydrogel, Ibuprofen, drug delivery.

\section{INTRODUCTION}

Among the diverse approaches that are possible for modifying polysaccharides, grafting of synthetic polymer is a convenient method to add new properties to a polysaccharide with minimum loss of the initial properties of the substrate. Graft copolymerization of vinyl monomers onto polysaccharides using free radical initiation has attracted the interest of many scientists. Up to now, considerable works have been devoted to the grafting of vinyl monomers onto the substrates, specially cellulose, Of the monomers grafted, acrylonitrile (MAN) has been the most frequently used one, mainly due to its highest grafting efficiency ${ }^{2-3}$, improving the thermal resistance of the graft copolymer and also the subsequent alkaline hydrolysis of the grafting product to obtain water absorbents .

This article represents synthesis of a novel superabsorbent hydrogel based on alginate-gpolyacrylamide for control delivery system. To the best of our knowledge based on a precise survey of the Chemical Abstracts, the present paper is the first report on the preparation of a super absorbing hydrogel through graft copolymerization of a vinyl monomer onto alginate.

\section{MATERIALS AND METHODS}

\section{Materials}

Sodium alginate (chemical grade, MW 50000 and follow chemical stracture) was purchased from Merck Chemical Co. (Germany). Ammonium persulfate (APS, from Fluka) and acrylamide (AAm, Rotterdam, the Netherlands), were of analytical grade and were used as received. All other chemicals were of analytical grade.

\section{Synthesis procedure of the hydrogel}

A general one step preparative method for synthesis of alginate-poly (acrylamide) hydrogel was conducted as follows. alginate $(0.50-1.50 \mathrm{~g})$ was added to a three-neck reactor equipped with a mechanical stirrer (Heidolph RZR 2021, three blade propeller type, $300 \mathrm{rpm}$ ), including $40 \mathrm{~mL}$ doubly distilled water. The reactor was immersed in a thermostated water bath preset at desired temperature $\left(35-70{ }^{\circ} \mathrm{C}\right)$. Then, at this temperature, 
APS was added and after stirring for $30 \mathrm{~min}$ (induction period) and a definite amount of acrylamide $(0.5-4.5 \mathrm{~mL})$ were added into the mixture. After a prescribed time (30-120 $\mathrm{min})$, the obtained hydrogel was poured into methanol (200 $\mathrm{mL}$ ) to dewater for $24 \mathrm{~h}$. Then, the product was filtered and dried in an oven at $60^{\circ} \mathrm{C}$ to reach a constant weight. The product was stored away from moisture, heat and light.

\section{Drug Loading Efficiency and In vitro Drug Release}

Powdered samples ( $1 \mathrm{~g} \pm 0.0001)$, with average particle sizes between 40-60 mesh (250$420 \mu \mathrm{m}$ ), were accurately weighted and immersed in an alkaline solution of ibuprofen (IBU, $0.54 \mathrm{~g}$ dissolved in $50 \mathrm{~mL}$ distilled water) at $0^{\circ} \mathrm{C}$ for $25 \mathrm{~h}$. The swollen hydrogels loaded with drug were placed in a vacuum oven and dried under vacuum at $37^{\circ} \mathrm{C}$. The loading amount of drug in the hydrogels was calculated from the decrease in the concentration of the IBU solution which was determined using a UV spectrophotometer (UV1201, Shimadzu, Kyoto, Japan). The loading efficiency of the alginate-based hydrogels was calculated as the ratio of the final to the initial IBU concentration.

In vitro release was carried out in duplicate by incubating $0.01 \pm 0.0001 \mathrm{~g}$ of the IBU-loaded hydrogels into a cellophane membrane dialysis bag $\left(D_{9402}\right.$, SIGMA-ALDRICH) in $50 \mathrm{~mL}$ of buffer solution (either $\mathrm{pH} 3$ or 9 ) at $37^{\circ} \mathrm{C}$. At specific time intervals, $1 \mathrm{~mL}$ aliquots of sample was withdrawn and after suitable dilution the concentration of drug released was measured by UV spectrophotometer. The drug release percent was calculated twice using the following equation:

$$
\text { Released drug }(\%)=R_{t} / L \times 100
$$

where $L$ and $R_{t}$ represent the initial amount of drug loaded and the final amount of drug released at time $\mathrm{t}$.

\section{RESULTS AND DISCUSSION}

\section{Synthesis and mechanism aspects}

A crosslinking graft copolymerization of acrylamide (AAm) onto alginate was conducted using ammonium persulfate (APS) as a water soluble initiator. The persulfate initiator is decomposed under heating to generate sulfate anion-radical (Scheme1). The radical abstracts hydrogen from the hydroxyl group of the polysaccharide substrate to form alkoxy radicals on the substrate. So, this persulfate-saccharide redox system is resulted in active centers on the substrate to radically initiate polymerization of AAm led to a graft copolymer. Since a crosslinking agent, e.g. MBA, is presented in the system, the copolymer comprises a crosslinked structure. It should be pointed out that the sulfate ion-radical may also initiate AAm homopolymerization. To minimize this undesired reaction, an ${ }^{2}$ induction period ${ }^{2}$ was provided in the synthesis, i.e. the initiator was introduced to the substrate before adding the monomer (See Experimental). Our preliminary

$$
\begin{aligned}
& \left(\mathrm{NH}_{4}\right)_{2} \mathrm{~S}_{2} \mathrm{O}_{3} \stackrel{70 \mathrm{C}}{\longrightarrow} 2 \mathrm{~S}_{4}^{\circ}+2 \stackrel{+}{\longrightarrow} \cdot \mathrm{I}_{4}
\end{aligned}
$$

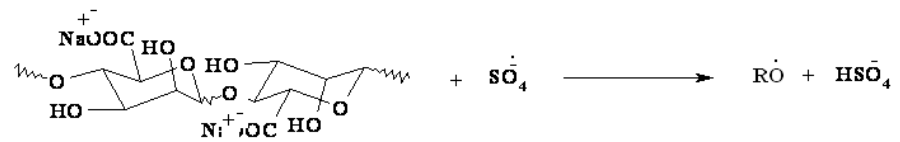

$$
\begin{aligned}
& \text { Alg }(\mathrm{ROH})
\end{aligned}
$$

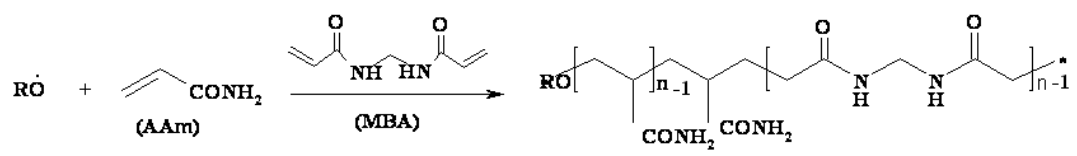

$$
\begin{aligned}
& \text { crosslinked H-Alg-g-PAAm }
\end{aligned}
$$

Scheme 1: Proposed mechanism for crosslinking of the H-Alg-g-PAAm hydrogel 
studied showed low homopolymer formation (less than $4 \%$ ) when the reaction was performed in the absence of crosslinker. In the presence of the crosslinker, however, the monomers are probably more intensely involved in the copolymeric network. Besides, the probable crosslinked hydrophilic homopolymer does not cause appreciable unwanted effects on absorbing properties of the final products ${ }^{5-8}$.

\section{Spectral characterization}

The grafting was confirmed by comparing the FTIR spectra of the polysaccharide substrate with that of the grafted products. Figure 1 shows the FTIR spectra of non-modified alginate and $\mathrm{H}$ alginate-g-PolyAAm. The broad band at 3200-3400 $\mathrm{cm}^{-1}$ is due to stretching of $-\mathrm{OH}$ groups of alginate. The IR spectrum of the $\mathrm{H}$-alginate-g-PolyAAm

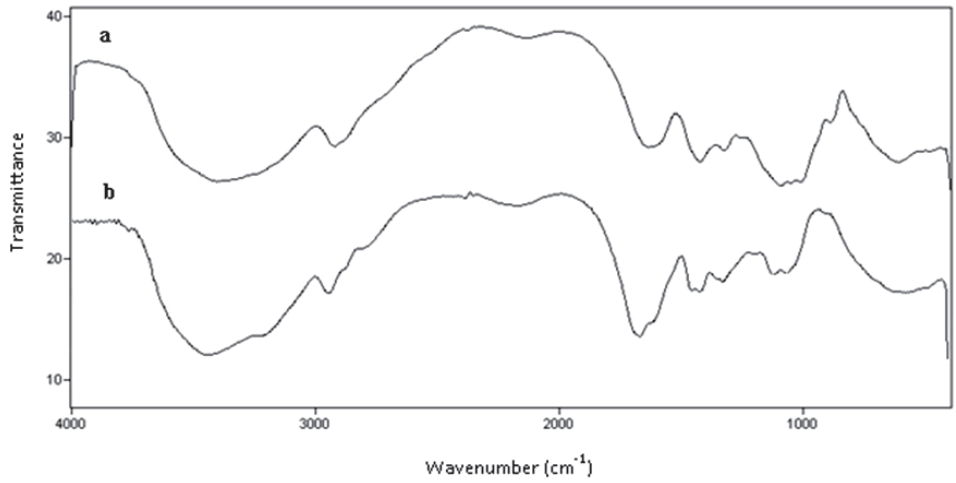

Fig. 1: FTIR spectra of alginate (a), $\mathrm{H}$-alginate-g-PolyAAm hydrogel(b)
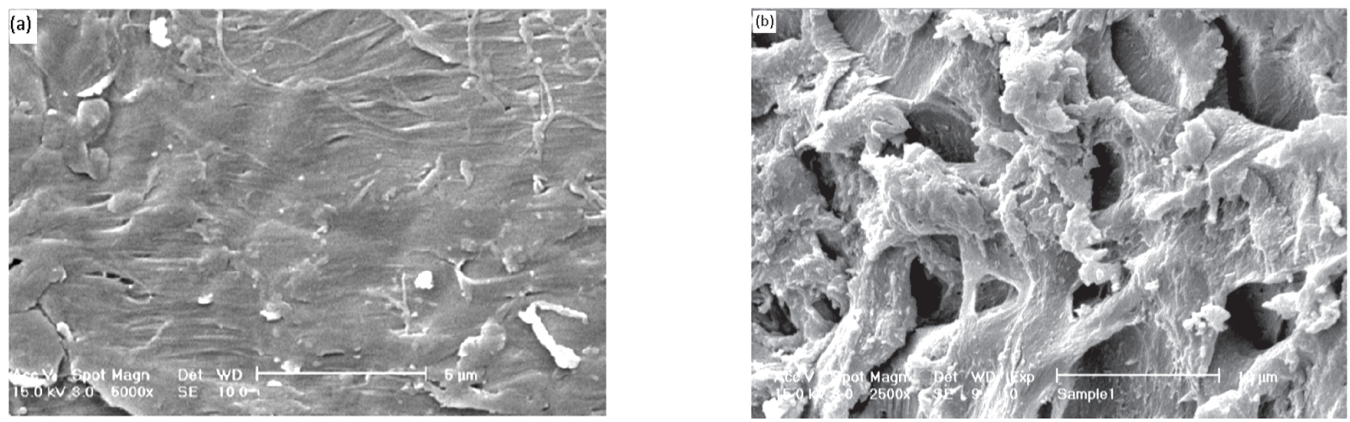

Fig. 2: SEM photograph of the(a) Surface of pure alginate;

(b) Surface of porous $\mathrm{H}$-alginate-g-PolyAAm hydrogel

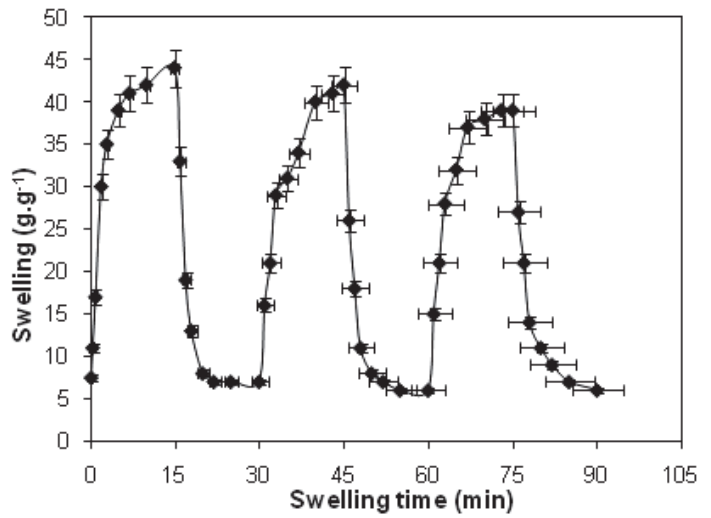

Fig. 3: On-off switching behavior as reversible pulsatile swelling ( $\mathrm{pH}$ 9.0) and deswelling (pH 3.0) of $\mathrm{H}$-alginate-g-PolyAAm hydrogel

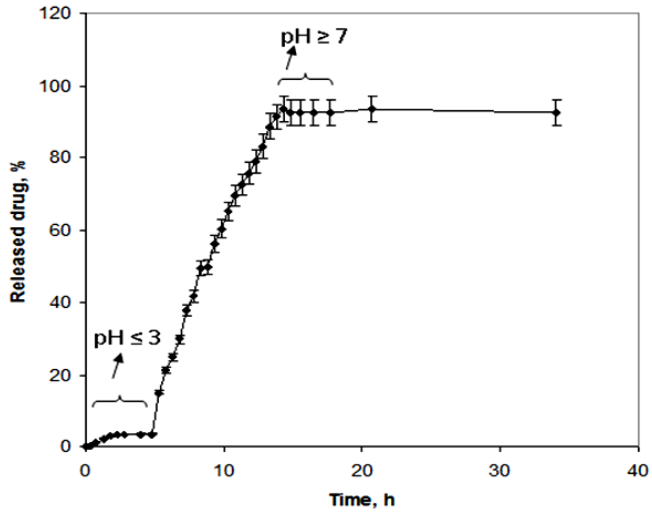

Fig. 4: Release of IBU from hydrogel carrier as a function of time and $\mathrm{pH}$ at $37^{\circ} \mathrm{C}$ 
hydrogel (Fig. 1(b)) shows a new characteristic absorption band at $1680 \mathrm{~cm}^{-1}$ verifying the formation of alginate-g-PAAm. This peak attributed to $\mathrm{C}=\mathrm{O}$ stretching in carboxamide functional groups of $\mathrm{PAAm}^{12-13}$. The stretching band of $-\mathrm{NH}$ overlapped with the $\mathrm{OH}$ stretching band of the alginate portion of the copolymer

\section{Scanning electron microscopy}

One of the most important properties that must be considered is hydrogel microstructure morphologies. Figure 2 shows the scanning electron microscope (SEM) photographs of the alginate surface (Fig. 2a) and surface of the $\mathrm{H}$ alginate-g-PolyAAm hydrogel(Fig. 2b). These pictures verify that the synthesized polymer in this work have a porous structure, where the pores might be induced into the hydrogel by water evaporation resulting from reaction heat. It is supposed that these pores are the regions of water permeation and interaction sites of external stimuli with the hydrophilic groups of the graft copolymers.

\section{pH-Reversibility for H-alginate-g-PolyAAm hydrogel}

Since the hydrogels show different swelling behavior at various $\mathrm{pHs}$, we investigated their $\mathrm{pH}$-reversibility in solutions buffered at $\mathrm{pH} 3.0$ and 9.0. A stepwise reproducible in swelling change of the hydrogel at $25^{\circ} \mathrm{C}$ with alternating $\mathrm{pH}$ between 3.0 and 9.0 is seen in Figure 3. At pH 9.0, the hydrogel swelled up to $43 \mathrm{~g} / \mathrm{g}$ due to anion-anion repulsive electrostatic forces, while, at $\mathrm{pH} 3.0$, it shrunk within a few minutes due to protonation of carboxylate groups. This sharp swelling-deswelling behavior of the hydrogels makes them suitable candidates for controlled drug delivery systems. Such on-off switching behavior as reversible swelling and deswelling has been reported for other ionic hydrogels ${ }^{11-12}$.
In vitro IBU Release in the Simulated Human Gastrointestinal System

To determine the potential application of alginate-based superabsorbent containing a pharmaceutically active compound, we have investigated the drug release behavior IBU form this system under physiological conditions. The percent of released drug from the polymeric carriers as a function of time is shown in Figure 4. The concentration of IBU released at selected time intervals was determined by UV spectrophotometer. The IBU-loaded hydrogels with high degrees of drug loading (>92\%) were prepared by the swellingdiffusion method. The amount of IBU released in a specified time from the $\mathrm{H}$-alginate-g-PolyAAm hydrogel decreased as the $\mathrm{pH}$ of the dissolution medium was lowered, indicating better release in a medium with a pH much higher than that of the stomach $^{11}$.

\section{CONCLUSIONS}

A novel superabsorbent hydrogel was synthesized via Graft copolymerization of acrylamide (AAm) onto alginate in an aqueous medium using a persulfate initiator. The synthesized hydrogel, $\mathrm{H}$-alginate-g-PolyAAm, exhibited high absorbency in aqueous solution.

The superabsorbent hydrogels exhibited also high sensitivity to $\mathrm{pH}$, so that, the reversible swelling-deswelling behavior in solutions with acidic and basic $\mathrm{pH}$, contributes to the suitability of these hydrogels as candidates for controlled drug delivery systems. In vitro drug-release studies in different buffer solutions showed that the most important parameter affecting the drug-release behavior of hydrogels is the $\mathrm{pH}$ of the solution.

\section{REFERENCES}

1. Buchholz, F.L. and Graham, A.T.. Modern Superabsorbent Polymer Technology; Wiley, New York ,(1997).

2. Yazdani-Pedram, M.; Retuert, J.; Quijada, R. Macromol Chem Phys, 201: 923 (2000).

3. Sugahara, Y.; Takahisa, O. J Appl Polym Sci, 82: 1437(2001).
4. Patel, G. M.; Trivedi, H. C. Eur Polym J, 35: 201(1999).

5. Silong, S.; Rahman, L. J Appl Polym Sci, 76: 516(2000).

6. Kost, J. In Encyclopedia of Controlled Drug Delivery; Mathiowitz, E., Ed.; Wiley: New York, 1: 445(1999). 
7. Po, R.. Water-absorbent Polymers, A Patent Survey, J. Macromol. Sci., Rev. Macromol. Chem. Phys., C34: 07-661(1994).

8. Kost, J. Encyclopedia of Controlled Drug Delivery. E. Mathiowitz (Ed.) Wiley, New York 1: 445 (1999).

9. Hoffman, A. S. Polymeric Materials Encyclopedia; Salamone, J. C.; Ed.; CRC Press, Boca Raton, FL. 5: 3282(1996).
10. Peppas, N.A. and Mikes, A.GHydrogels in Medicine and Pharmacy, CRC Press Inc., Boca Raton, Florida., 1: 27(1986).

11. Gan, L.H., Deen, G.R., Gan, Y.Y. and Tam, K.C. Eur. Polym. J., 37: 1473-1478 (2001).

12. Mahkam, M., Doostie, L. and Siadat, S.O.R.. Inflammo Pharmacology.,14: 72-75(2006).

13. Mahkam, M. and Allahverdipoor, M.. Drug Targeting, 12: 151-156 (2004). 